\title{
Indiskrete Logarithmen?
}

\author{
Jens Zumbrägel
}

\begin{abstract}
Die moderne Public-Key-Kryptographie, begründet durch die bahnbrechende Arbeit von Diffie und Hellman, ist seit jeher mit der Schwierigkeit des diskreten Logarithmusproblems verbunden. Allerdings ist in Körpern kleiner Charakteristik die Hartnäckigkeit dieses Problems nicht so hoch, wie lange Zeit angenommen. Daraus resultieren beträchtliche Rekordberechnungen, sowie Konsequenzen für die Sicherheit einiger Kryptosysteme.
\end{abstract}

Die Kryptographie, die Kunst des geheimen Schreibens, ist etwa so alt wie die Verbreitung der Schrift selbst, ihre Geschichte so spannend wie aufschlussreich. Die Idee der Public-Key-Kryptographie revolutionierte das Verständnis von Verschlüsselungs- und Signatursystemen grundlegend und ermöglichte die moderne Kryptographie der heutigen Informationsgesellschaft.

Die nunmehr angewandten Public-Key-Kryptoverfahren beruhen im Wesentlichen auf zwei algorithmischen Problemen, dem diskreten Logarithmusproblem und dem Faktorisierungsproblem. Beide sind seit langem untersucht und finden als Basis der Kryptosysteme von Diffie und Hellman (DH) bzw. von Rivest, Shamir und Adleman (RSA) Eingang in unseren Alltag.

\section{Das diskrete Logarithmusproblem}

Erste Vorarbeiten zum diskreten Logarithmusproblem finden sich bereits bei Euler und Gauß. Euler zeigte, dass für jede Primzahl $p$ eine sogenannte Primitivwurzel existiert, d.h. eine Zahl $g$ derart, dass $g^{x} \bmod p$ alle Zahlen in $\{1, \ldots, p-1\}$ trifft wenn $x$ die ganzen Zahlen durchläuft. $\mathrm{Zu}$ einer gegebenen Zahl $h$ zwischen 1 und $p-1$ wird die kleinste natürliche Zahl $x$ mit $h \equiv g^{x}$ mod $p$ von Gauß als "Index “ bezeichnet und ist heute als diskreter Logarithmus bekannt (siehe Tabelle 1 als Beispiel).

Einen natürlichen Kontext des diskreten Logarithmusproblems stellen endliche zyklische Gruppen dar, also Gruppen die von einem Element erzeugt werden. Es sei $G$ eine solche Gruppe und $g \in G$ ein Erzeuger, was bedeutet, dass sich jedes Gruppenelement $h \in G$ als eine Potenz

$$
h=g^{x}=\underbrace{g \cdot \ldots \cdot g}_{x \mathrm{mal}}
$$

für eine natürliche Zahl $x$ schreiben lässt. Die Zahl $x$ ist dabei eindeutig modulo der Gruppenordnung $n:=|G|$ und wird als diskreter Logarithmus $\log _{g} h:=x$ bezeichnet. Wie bei der klassischen Exponentialfunktion ist also der diskrete Logarithmus die Umkehrabbildung der diskreten Potenzierung

$$
f: \mathbb{Z}_{n} \rightarrow G, \quad x \mapsto g^{x},
$$

wobei $\mathbb{Z}_{n}=\mathbb{Z} / n \mathbb{Z}$ die Restklassengruppe modulo $n$ bezeichne. Wird insbesondere für eine Primzahl $p$ die „prime Restklassengruppe“ als multiplikative Gruppe $G=\left(\mathbb{Z}_{p}\right)^{*}$ der Ordnung $n=p-1$ betrachtet, so entsprechen die Primitivwurzeln gerade den Erzeugern dieser (zyklischen) Gruppe.

\section{Einbahnfunktionen}

Die diskrete Exponentialfunktion $f$ hat eine bemerkenswerte Eigenschaft, nämlich die einer Einbahnfunktion: Es ist (auch für sehr große Gruppenordnung $n$ ) leicht, Funktionswerte $f(x)$ zu gegebenen Argumenten $x \in \mathbb{Z}_{n}$ zu berechnen, allerdings äußerst schwierig, die Umkehrabbildung auszuwerten (siehe Abbildung 1).

Der Begriff eines „rechnerisch leichten“ Problems wird dabei so formalisiert, dass es einen Algorithmus gibt, der polynomielle Zeit in der Eingabelänge $\log n$ benötigt. Im Fall der Potenzierung ist dies durch iteriertes Quadrieren und Multiplizieren gemäß der Binärdarstellung des Exponenten möglich; zum Beispiel ist

$$
a^{13}=a^{8} a^{4} a=\left(\left(a^{2} a\right)^{2}\right)^{2} a .
$$

Die Auswertung der Umkehrfunktion der diskreten Exponentialfunktion wird als diskretes Logarithmusproblem bezeichnet. Hierfür ist kein polynomieller Algorithmus bekannt, weswegen das diskrete Logarithmusproblem allgemein als schwierig angesehen wird.

Diese Einbahneigenschaft tritt auch bei kryptographischen Hashfunktionen auf (welche allerdings nicht bijektiv

Tabelle 1. Diskrete Logarithmen modulo $p=13$ mit $g=2$. Der diskrete Logarithmus von $h=5$ mod 13 ist $x=9$.

\begin{tabular}{c|cccccccccccc}
$h=g^{x}$ & 1 & 2 & 4 & 8 & 3 & 6 & 12 & 11 & 9 & 5 & 10 & 7 \\
\hline$x$ & 0 & 1 & 2 & 3 & 4 & 5 & 6 & 7 & 8 & 9 & 10 & 11
\end{tabular}




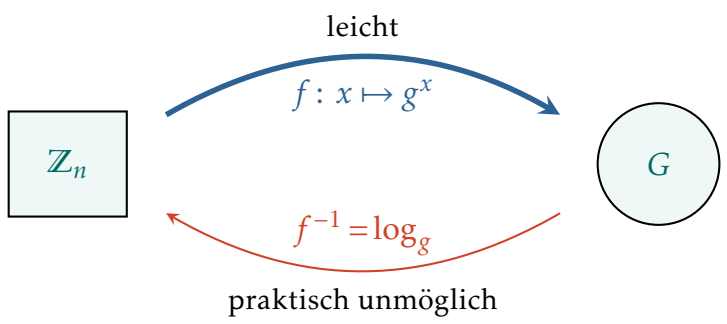

Abbildung 1. Diskrete Potenzierung in einer Gruppe $G$ als Einbahnfunktion

sind). So beruht etwa die Sicherheit von Kryptowährungen wie Bitcoin auf der Schwierigkeit, Argumente mit vorgegebenem Hashwert zu finden.

\section{Die Idee der Public-Key-Kryptographie}

Historische Geheimhaltungsverfahren, von Cäsars einfacher Substitutionschiffre bis zur im zweiten Weltkrieg eingesetzten Enigma-Maschine, sind aus heutiger Sicht der symmetrischen Kryptographie zuzuordnen: Die Sicherheit hängt entscheidend von der Geheimhaltung eines Schlüsselcodes $a b$, welcher sowohl für die Verschlüsselung als auch für die Entschlüsselung von Nachrichten benutzt wird. Dies ließ die Frage nach einem sicheren Schlüsselaustausch und einem effizienten Schlüsselmanagement in größeren Kommunikationsnetzwerken unbeantwortet.

Die von Diffie und Hellman in ihrer bahnbrechenden Arbeit [1] vorgeschlagene Public-Key-Kryptographie revolutionierte diesen Ansatz: Jede Partei besitzt nun zwei Schlüsselcodes, wobei ein Schlüssel zum Verschlüsseln von Nachrichten offen gelegt wird (public key) und nur der andere Schlüssel zum Entschlüsseln geheim bleibt (private key). So können Nachrichten zwischen beliebigen Teilnehmenden verschlüsselt und entschlüsselt werden, ohne dass für jedes Paar ein eigener Schlüssel vereinbart werden muss. Diffie und Hellman schlugen kein konkretes Public-KeyVerfahren vor, doch wurde kurze Zeit später von Rivest, Shamir und Adleman das berühmte RSA-Verfahren publi-

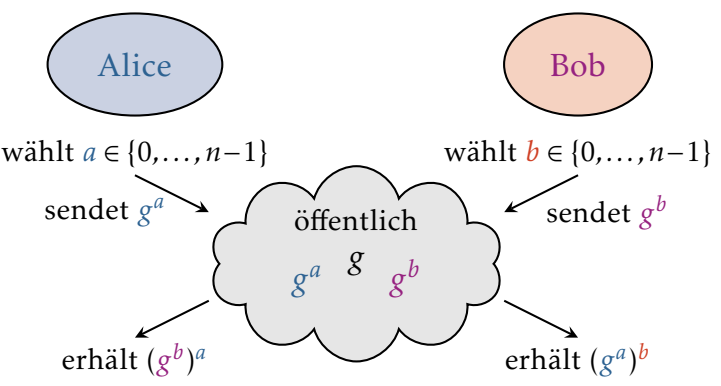

Der vereinbarte Schlüssel ist $\left(g^{b}\right)^{a}=g^{a b}=\left(g^{a}\right)^{b}$.

Abbildung 2. Diffie-Hellman-Protokoll in einer Gruppe G mit Erzeuger $g$ ziert [7], welches auf dem Problem der Zahlfaktorisierung basiert.

In der Arbeit von Diffie und Hellman befindet sich jedoch ein Protokoll, welches einen Schlüsselaustausch zwischen zwei Parteien über einen unsicheren öffentlichen Kanal ermöglicht. Es beruht auf dem diskreten Logarithmusproblem und wurde später zu Public-Key-Verfahren für Verschlüsselung und digitale Signatur erweitert [2]. Heute wird es beispielsweise im Internet beim Aufruf von HTTPSSeiten verwendet.

Das Diffie-Hellman-Protokoll

Beim Diffie-Hellman-Schlüsselaustausch einigen sich die Parteien „Alice“ und „Bob“ zunächst auf eine Gruppe G und einen Erzeuger $g \in G$. Bezeichne wieder $n=|G|$ die Gruppenordnung, so wählen Alice und Bob jeweils zufällig geheime Exponenten $a, b \in\{0, \ldots, n-1\}$ und senden die Potenzen $g^{a}, g^{b} \in G$ über einen öffentlichen Kanal, wodurch sie mit der empfangenen Information der anderen Partei jeweils einen gemeinsamen Schlüssel $g^{a b}$ berechnen können (siehe Abbildung 2 sowie Abbildung 3 als Beispiel in $G=\left(\mathbb{Z}_{13}\right)^{*}$.)

Die Sicherheit des Diffie-Hellman-Protokolls hängt offenbar eng mit dem diskreten Logarithmusproblem zusammen, denn wenn dieses gelöst wird, so kann der vereinbarte Schlüssel aus den öffentlichen Daten rekonstruiert werden.

Es sei angemerkt, dass die Schwierigkeit des diskreten Logarithmusproblems zwar notwendig, aber nicht unbedingt hinreichend für die Sicherheit des Protokolls ist. Denn
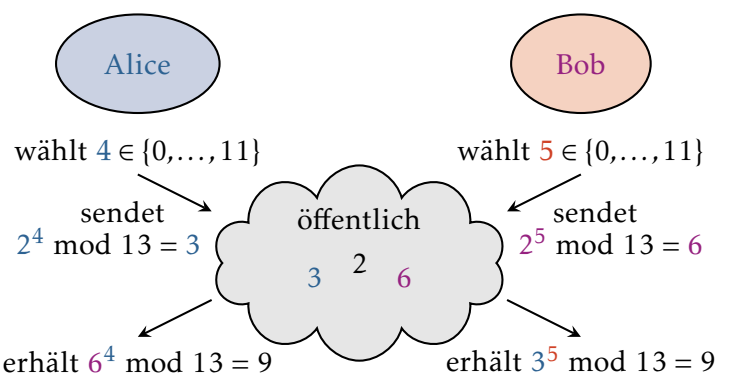

Abbildung 3. Beispiel eines Diffie-Hellman-Protokolls in der Gruppe $\left(\mathbb{Z}_{13}\right)^{*}$ mit Erzeuger $2 \bmod 13$ 
zum einen ist es denkbar, dass der gemeinsame Schlüssel $g^{a b}$ aus der Information von $g, g^{a}, g^{b}$ gewonnen werden könnte, ohne einen diskreten Logarithmus zu berechnen. Andererseits besteht ohne Authentisierungsmaßnahmen die Gefahr, dass sich eine betrügerische dritte Partei zwischen Alice und Bob schaltet. Diese Angriffe wollen wir hier jedoch außer Acht lassen und uns auf das diskrete Logarithmusproblem konzentrieren.

\section{Die Wahl der Gruppe}

Ein Vorteil der DH-Schlüsselvereinbarung (und den daraus abgeleiteten Verschlüsselungs- und Signaturverfahren) gegenüber RSA ist, dass es durch die Wahl der Gruppe eine größere Flexibilität erlaubt. Neben der primen Restklassengruppe $\left(\mathbb{Z}_{p}\right)^{*}$ verwendet man insbesondere die Einheitengruppen von endlichen Körpern, sowie die Gruppe der rationalen Punkte von elliptischen Kurven über endlichen Körpern.

Bereits frühzeitig sind „binäre Körper“ $\mathbb{F}_{2^{m}}$ und deren Einheitengruppen $G=\left(\mathbb{F}_{2^{m}}\right)^{*}$ ins Spiel gebracht worden, unter anderem weil diese für Computer sehr effiziente Operationen erlauben. Binäre Körper zählen zu den endlichen Körpern kleiner Charakteristik, welche wir im Folgenden betrachten wollen. Denn für diesen Fall gibt es dramatische neuere Entwicklungen bei der Einschätzung des diskreten Logarithmusproblems.

\section{Körper kleiner Charakteristik}

Für jede Primzahlpotenz $q=p^{m}$ existiert eindeutig ein Körper mit $q$ Elementen, welchen wir mit $\mathbb{F}_{q}$ bezeichnen. Wenn $q=p$ eine Primzahl ist, so handelt es sich um den Primkörper $\mathbb{Z}_{p}=\mathbb{Z} / p \mathbb{Z}$. Im allgemein Fall betrachtet man Polynome über einem Primkörper modulo einem irreduziblen Polynom $f \in \mathbb{Z}_{p}[X]$ von Grad $m$, also

$$
\mathbb{F}_{q}=\mathbb{Z}_{p}[X] / f \mathbb{Z}_{p}[X]
$$

Elemente in $\mathbb{F}_{q}$ werden dabei als Polynome in $x$ von Grad kleiner $m$ repräsentiert (wobei $x$ die Klasse von $X$ modulo $f$ sei).

Sei beispielsweise $q=4=2^{2}$, dann gibt es den Körper $\mathbb{F}_{4}=\mathbb{Z}_{2}[X] / f \mathbb{Z}_{2}[X]$ mit $f:=X^{2}+X+1$, und wir haben die Repräsentanten $\mathbb{F}_{4}=\{0,1, x, x+1\}$ mit den Verknüpfungstafeln aus Tabelle 2.

Tabelle 2. Verknüpfungen des Körpers $\mathbb{F}_{4}$. Es gilt etwa $x \cdot x=x+1$ in $\mathbb{F}_{4}$, $\operatorname{denn} X^{2} \equiv X+1 \bmod f$.

\begin{tabular}{c|cccc}
+ & 0 & 1 & $x$ & $x+1$ \\
\hline 0 & 0 & 1 & $x$ & $x+1$ \\
1 & 1 & 0 & $x+1$ & $x$ \\
$x$ & $x$ & $x+1$ & 0 & 1 \\
$x+1$ & $x+1$ & $x$ & 1 & 0 \\
$\cdot$ & 0 & 1 & $x$ & $x+1$ \\
\hline 0 & 0 & 0 & 0 & 0 \\
1 & 0 & 1 & $x$ & $x+1$ \\
$x$ & 0 & $x$ & $x+1$ & 1 \\
$x+1$ & 0 & $x+1$ & 1 & $x$
\end{tabular}

Tabelle 3. Diskrete Logarithmen in $\mathbb{F}_{8}=\mathbb{Z}_{2}[X] / f \mathbb{Z}_{2}[X]$ mit $f:=$ $X^{3}+X+1$ und Erzeuger $g:=x \in\left(\mathbb{F}_{8}\right)^{*}$

$$
\begin{array}{c|ccccccc}
h=g^{x} & 1 & x & x^{2} & x+1 & x^{2}+x & x^{2}+x+1 & x^{2}+1 \\
\hline x & 0 & 1 & 2 & 3 & 4 & 5 & 6
\end{array}
$$

Wie im Fall der primen Restklassengruppe $\left(\mathbb{Z}_{p}\right)^{*}$ ist für endliche Körper $\mathbb{F}_{q}$ die Einheitengruppe $\left(\mathbb{F}_{q}\right)^{*}=\mathbb{F}_{q} \backslash\{0\}$ eine zyklische Gruppe (der Ordnung $n=q-1$ ). Somit lässt sich auch für diesen Fall bezüglich eines Erzeugers $g \in\left(\mathbb{F}_{q}\right)^{*}$ der diskrete Logarithmus $\log _{g} h$ von $h \in\left(\mathbb{F}_{q}\right)^{*}$ definieren als die natürliche Zahl $z$ (eindeutig modulo $n$ ) mit $h=g^{z}$ (siehe Tabelle 3 als Beispiel).

Bei einem Körper mit $q=p^{m}$ Elementen wird die Primzahl $p$ als seine Charakteristik bezeichnet. Ist diese polynomiell in $\log q$ bzw. sogar konstant, so sprechen wir von (einer Familie von) Körpern kleiner Charakteristik.

\section{Rekordberechnungen}

Eine gewisse Tradition ist es, die Effizienz von Algorithmen, deren hochoptimierte Implementierungen und die Leistungsfähigkeit aktueller Hardware durch zahlentheoretische Großberechnungen nahe der Machbarkeitsgrenze zu illustrieren. Beispielsweise finden sich Rekorde über die größten ,bekannten“ Primzahlen, oft in der Mersenne-Form $n=2^{m}-1$. So lautet die größte so gefundene und damit bekannte Primzahl 277232917-1, gefunden im Dezember 2017, vgl. www.mersenne.org.

Tabelle 4. Algorithmen und Rekordberechnungen für diskrete Logarithmen in $\left(\mathbb{F}_{q}\right)^{*}$, wobei $q=p^{m}$, Laufzeit $L(a, c):=2^{c \cdot b^{a} \cdot\left(\log _{2} b\right)^{1-a}}$ mit $b=\log _{2} q$ (Quelle: NMBRTHRY Mailing List, siehe auch en.wikipedia. org/wiki/Discrete_logarithm_records)

\begin{tabular}{rcll}
\hline Bitl. $b$ & Char. $p$ & Wer und wann & Laufzeit \\
\hline 127 & 2 & Coppersmith 1984 & $L\left(\frac{1}{3}, 1.587\right)$ \\
401 & 2 & Gordon, McCurley 1992 & $L\left(\frac{1}{3}, 1.587\right)$ \\
n/a & klein & Adleman 1994 & $L\left(\frac{1}{3}, 1.923\right)$ \\
427 & groß & Weber, Denny 1998 & $L\left(\frac{1}{3}, 1.526\right)$ \\
521 & 2 & Joux, Lercier 2001 & $L\left(\frac{1}{3}, 1.526\right)$ \\
607 & 2 & Thomé 2001 & $L\left(\frac{1}{3}, 1.587\right)$ \\
613 & 2 & Joux, Lercier 2005 & $L\left(\frac{1}{3}, 1.526\right)$ \\
556 & mittel & Joux, Lercier 2006 & $L\left(\frac{1}{3}, 1.442\right)$ \\
676 & 3 & Hayashi et al. 2010 & $L\left(\frac{1}{3}, 1.442\right)$ \\
923 & 3 & Hayashi et al. 2012 & $L\left(\frac{1}{3}, 1.442\right)$ \\
1175 & medium & Joux 24 Dez 2012 & $L\left(\frac{1}{3}, 1.260\right)$ \\
1425 & medium & Joux 6 Jan 2013 & $L\left(\frac{1}{3}, 1.260\right)$ \\
1778 & 2 & Joux 11 Feb 2013 & $L\left(\frac{1}{4}+o(1)\right)$ \\
1971 & 2 & GGMZ 19 Feb 2013 & $L\left(\frac{1}{3}, 0.763\right)$ \\
4080 & 2 & Joux 22 Mär 2013 & $L\left(\frac{1}{4}+o(1)\right)$ \\
6120 & 2 & GGMZ 11 Apr 2013 & $L\left(\frac{1}{4}\right)$ \\
6168 & 2 & Joux 21 Mai 2013 & $L\left(\frac{1}{4}+o(1)\right)$ \\
n/a & klein & BGJT 18 Jun 2013 & $L(0+o(1))$ \\
9234 & 2 & GKZ 31 Jan 2014 & $L\left(\frac{1}{4}+o(1)\right)$ \\
n/a & klein & GKZ o6 Jul 2015 & $L(0+o(1))$ \\
\hline & & &
\end{tabular}


Im Kontext der Public-Key-Kryptographie interessiert man sich naheliegender Weise für diejenigen zahltheoretischen Probleme, welche den Verfahren als Grundlage dienen. Die Kenntnis der jeweils größten Probleminstanzen, welche mit modernen High-Performance-Clustern gerade noch lösbar sind, hilft somit, die Sicherheitsparameter der Kryptoverfahren möglichst treffend zu bewerten.

Für das weitverbreitete RSA-Kryptoverfahren beispielsweise wurde lange Zeit propagiert, es sei ausreichend, die Sicherheit auf der Faktorisierung einer 1024-bit-Zahl zu basieren. Große Aufmerksamkeit erfuhr deshalb die erfolgreiche Faktorisierung einer 768-bit-Zahl im Dezember 2009 [6], ein Rekord der bis heute gültig ist. Insgesamt 13 Autoren arbeiteten an dieser Berechnung für mehr als zwei Jahre und verwendeten eine Rechenzeit von etwa 20 Millionen Core-Stunden.

Bei den DH-basierten Kryptosystemen interessiert man sich entsprechend für die Berechnung eines diskreten Logarithmus in einer größtmöglichen Gruppe, wobei diese beispielsweise von einem endlichen Körper oder einer elliptischen Kurve kommen kann. Viele Rekordberechnungen treten bei endlichen Körpern auf und wir stellen einige
Meilensteine in Tabelle 4 zusammen. Die Aufstellung zeigt insbesondere, welche großen Fortschritte seit 2013 zu verzeichnen sind, sowohl auf algorithmischer Seite als auch bei der konkreten Bitlänge (Verzehnfachung seit 2012).

\section{Höhere Zerfallswahrscheinlichkeiten}

Was ist der Grund für diese dramatische Entwicklung der letzten Jahre im Fall der Körper kleiner Charakteristik? Dazu müssen wir das Prinzip der Indexkalkül-Methode verstehen, welches beim diskreten Logarithmusproblem in endlichen Körpern üblicherweise angewandt wird. Die Idee hierbei ist, zunächst alle diskreten Logarithmen für eine gewisse Teilmenge von „kleinen“ Gruppenelementen (der sogenannten Faktorbasis) zu berechnen, indem ein großes lineares Gleichungssystem aufgestellt und gelöst wird. Danach wird diese Information verwendet, um die Lösung einer gegebenen Logarithmusaufgabe zu finden. Eine grundlegende Frage für die Laufzeit von Indexkalkül-Algorithmen ist, wie effizient dieses lineare Gleichungssystem aufgestellt werden kann (Phase der Relationenerzeugung bzw. des „Siebens“).

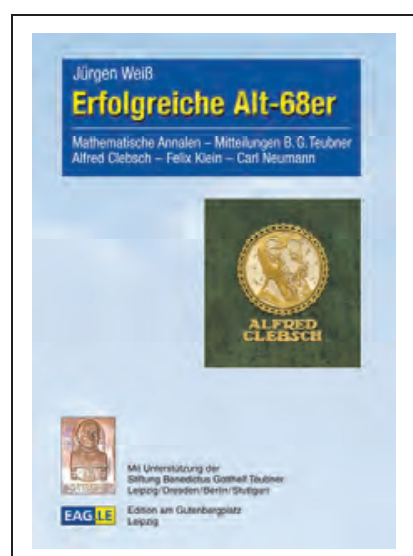

J. Weiß. EAGLE 101.

1. A. 2018. 978-3-95922-101-6

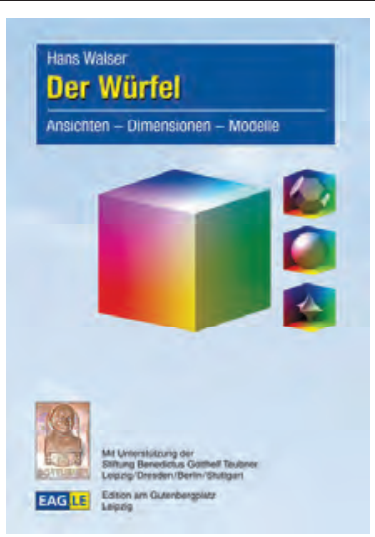

H. Walser. EAGLE 102.

1. A. 2018. $978-3-95922-102-3$

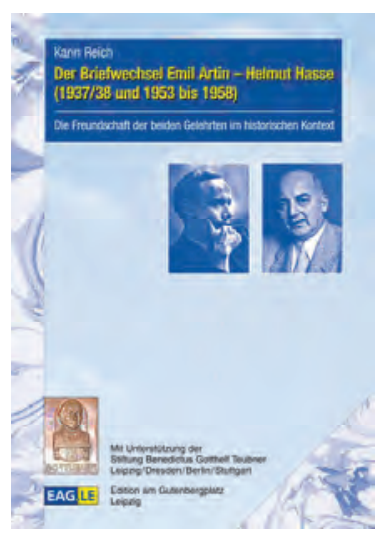

K. Reich. EAGLE 103.

1. A. 2018. $978-3-95922-103-0$

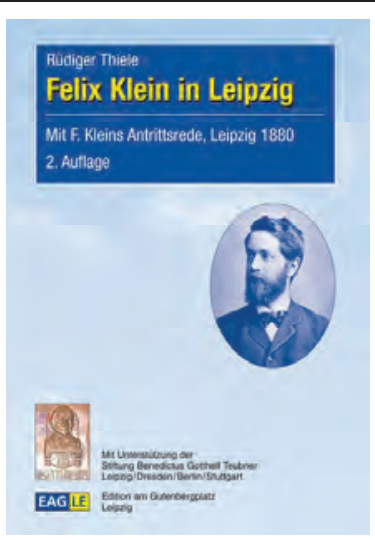

R. Thiele. EAGLE 047.

2. A. 2018. 978-3-95922-047-7

\section{EAG.LE}

Leipzig 1868: B. G. Teubners Mitteilungen erscheinen (regelmäßig bis 1914).

Leipzig 1868: Erstes Heft Mathematische Annalen (mit der Jahreszahl 1869) erscheint bei B. G. Teubner / seit 1920 bei J. Springer.

Leipzig 1868: Erster Brief von Felix Klein an B. G. Teubner (im Namen der Erben seines verstorbenen Lehrers Julius Plücker, Bonn).
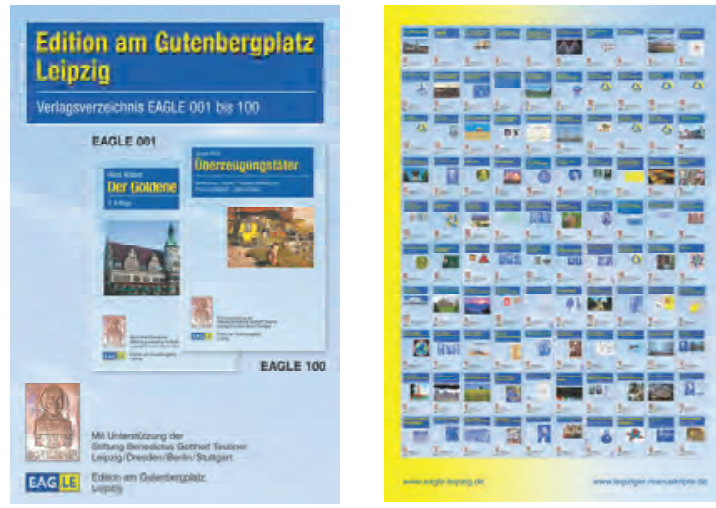

Edition am Gutenbergplatz Leipzig. Verlagsverzeichnis EAGLE 001-100: www.eagle-leipzig.de/verlagsverzeichnis-EAGLE-001-100.htm
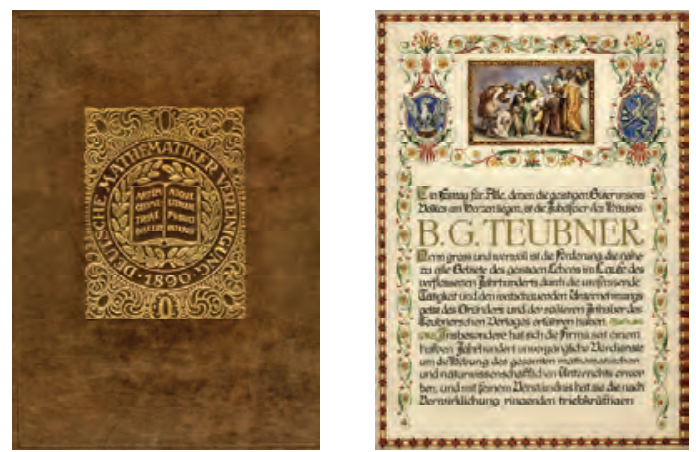

Gratulationsadressen 1911 (100 Jahre B. G. Teubner in Leipzig). Erstmals ganzseitig, farbig in: www.eagle-leipzig.de/101-weiss.htm Mit einem Beitrag von G. Ackermann, Berlin. Mit einem Geleitwort von J. Jost, Leipzig. 
Die effizientesten Siebeverfahren für Logarithmen in endlichen Körpern verwendeten bis 2012 jeweils ein ähnliches Prinzip. Im Fall kleiner (bzw. „mittlerer“) Charakteristik lässt sich dazu nach Joux und Lercier [4] das folgende kommutative Diagramm betrachten:

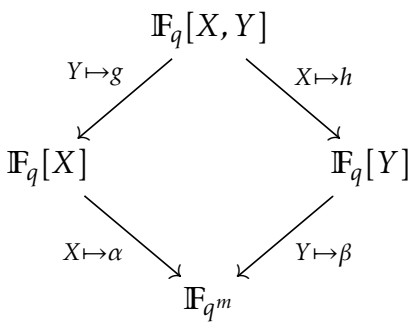

Dabei sind $g \in \mathbb{F}_{q}[X]$ und $h \in \mathbb{F}_{q}[Y]$ Polynome und $\alpha, \beta \in \mathbb{F}_{q^{m}}$ Körperelemente mit $\alpha=h(\beta)$ und $g(\alpha)=\beta$. Für $a, b, c \in \mathbb{F}_{q}$ sei nun $X Y+a Y+b X+c \in \mathbb{F}_{q}[X, Y]$ gegeben, also in $\mathbb{F}_{q^{m}}$ entlang des Diagramms

$$
\alpha g(\alpha)+a g(\alpha)+b \alpha+c=h(\beta) \beta+a \beta+b h(\beta)+c .
$$

Die Faktorbasis enthält alle linearen Polynome $X+u$ und $Y+v$ (bzw. $\alpha+u$ und $\beta+v$ ), wobei $u, v \in \mathbb{F}_{q}$. Eine Relation ist dann gefunden, wenn beide Seiten, d. h. $X g+a g+b X+c$ und $h Y+a Y+b h+c$ vollständig in Linearfaktoren zerfallen. Die Wahrscheinlichkeit, dass ein zufälliges Polynom von Grad $d$ zerfällt, ist etwa $\frac{1}{d !}$. Entsprechend können die Parameter $m, \operatorname{deg} g, \operatorname{deg} h$ so balanciert werden, dass eine $L\left(\frac{1}{3}\right)$-Laufzeit resultiert.

Entscheidend für die Revolution des diskreten Logarithmusproblems in kleiner Charakteristik ist nun eine einfache, ganz spezielle und überraschend unbalancierte Wahl der Parameter. Entgegen der gängigen Praxis, wonach Zerfallswahrscheinlichkeiten wie bei zufälligen Polynomen erwartet werden, führt folgende Festlegung zu einem ganz anderen Verhalten:

$$
g=X^{q}, \quad \operatorname{deg} h \leq 2 .
$$

Tatsächlich zerfällt die linke Seite $X^{q+1}+a X^{q}+b X+c$ dann über einem Erweiterungskörper $\mathbb{F}_{q^{k}}$ nicht mit einer Wahrscheinlichkeit von $\frac{1}{(q+1) !}$, sondern etwa $\frac{1}{q^{3}}$. Der prinzipielle Grund hierfür liegt in der systematischen Gleichung

$$
X^{q}-X=\prod_{\mu \in \mathbb{F}_{q}}(X-\mu),
$$

sowie deren Möbiustransformierte unter $X \mapsto \frac{\alpha X+\beta}{\gamma X+\delta}$ über $\mathbb{F}_{q^{k}}$. Es ist diese enorm hohe Zerfallswahrscheinlichkeit, die zu einer Relationenerzeugung in polynomieller Laufzeit führt!
Angemerkt sei allerdings, dass einige technische Schwierigkeiten verbleiben. Dazu gehört der Erweiterungsgrad $k$ und die Darstellung von $\mathbb{F}_{q^{m}}$ als Restklassenring $\mathbb{F}_{q}[X] / f \mathbb{F}_{q}[X]$ mit geeignetem irreduziblen Polynom $f$. Weiterhin erhöht sich die relative Schwierigkeit der letzten Phase des Indexkalküls, das Darstellen eines beliebigen Logarithmus mittels Faktorbasis-Logarithmen. Doch schließlich konnten bei fester Charakteristik in $\mathbb{F}_{p^{m}}$ Algorithmen mit quasipolynomieller Gesamtlaufzeit $m^{O(\log m)}$ gefunden werden. Mehr dazu findet sich in zwei kürzlich erschienenen Übersichtsartikeln $[3,5]$.

\section{Indiskrete Logarithmen? - kleiner Ausblick}

Ist bei den DH-basierten Kryptosystem die „Diskretion“ aufgrund des diskreten Logarithmusproblems nun verloren? Nicht ganz, denn Verfahren, die auf Primkörpern oder elliptischen Kurven basieren, gelten nach wie vor als sicher. Es scheint derzeit kaum möglich, die neuen Angriffe in Körpern kleiner Charakteristik auf Primkörper zu übertragen. Doch abgesehen von möglichen Angriffen durch Quantencomputer sind auch weitere Fortschritte bei klassischen Algorithmen für diskrete Logarithmen nicht auszuschließen. Die letzten Jahre haben gezeigt, dass stets überraschende Entwicklungen auf diesem Gebiet möglich sind. Es bleibt jedenfalls spannend!

\section{Literatur}

[1] W. Diffie, M. Hellman. New directions in cryptography. IEEE Trans. Inform. Theory 22.6 (1976), 644-654.

[2] T. ElGamal. A public key cryptosystem and a signature scheme based on discrete logarithms. IEEE Trans. Inform. Theory 31.4 (1985), 469-472.

[3] R. Granger, T. Kleinjung, J. Zumbrägel. Indiscreet logarithms in finite fields of small characteristic. Adv. Math. Comm. 12.2 (2018), 263-286.

[4] A. Joux, R. Lercier. The function field sieve in the medium prime case. In: Advances in Cryptology - EUROCRYPT 2006, LNCS 4004, Springer (2006), 254-270.

[5] A. Joux, A. Odlyzko, C. Pierrot. The past, evolving present, and future of the discrete logarithm. In: Open Problems Math. Comput. Science, Springer (2014), 5-36.

[6] T. Kleinjung et al. Factorization of a 768 -bit RSA modulus. In: Advances in Cryptology - CRYPTO 2010, LNCS 6223, Springer (2010), 333-350.

[7] R. L. Rivest, A. Shamir, L. Adleman. A method for obtaining digital signatures and public-key cryptosystems. Commun. ACM 21.2 (1978), 120-126. 